\title{
Reflexiones sobre la tuberculosis en Chile, 2006
}

\author{
MANUEL ZÚÑIGA G.*, PABLO MARCONE E.** y CECILIA RIQUELME J.***
}

\section{Reflections about Tuberculosis in Chile}

\section{Situación Epidemiológica}

En los años 2005 y 2006, el total de casos notificados de Tuberculosis en todas sus formas (TF), alcanzó a 2546 y 2362 (extrapolación de datos hasta el tercer trimestre del 2006), respectivamente. De éstos, 2316 y 2164 corresponden a casos nuevos ("incidencia") con tasas de 14,2 y $13,3 / 100.000$ habitantes ${ }^{1}$.

Para comprender la evolución de la tuberculosis en el tiempo, es necesario analizar la tendencia histórica de las tasas (Tabla 1). Las tasas extrapoladas para el año 2006: 14,4 del total de casos notificados TF (13,2 de casos nuevos TF y 6,3 de bacilíferos pulmonares), son las más bajas registradas en Chile.

El ritmo de declinación del total de casos notificados en el decenio 1995-2004, alcanzó a $-6,4 \%$, con lo cual cabía esperar tasas $\mathrm{TF}$ de 15,5 y de 14,5 para los años 2005 y 2006 . Las tasas de morbilidad (casos nuevos) correspondientes serían de 14,3 y de 13,3 respectivamente. Las tasas de morbilidad observada, de 14,2 y de 13,2 corresponden con gran exactitud a lo esperado de acuerdo a la tendencia.

Con los resultados alcanzados en los últimos dos años se ha recuperado el ritmo de declinación de la morbilidad por tuberculosis, superando así el período de estancamiento registrado con mayor claridad entre los años 2000 a 2003. La mayor preocupación derivó de la comprobación de diferencias entre dos períodos del decenio: entre 1995-2000 el coeficiente de declinación fue de $-7,7 \%$ anual; en cambio, entre el 2000-2005, éste se redujo al $-4,8 \%$ anualmente. Los resultados obtenidos en los últimos dos años, permiten enfrentar con mayor optimismo la po- sibilidad de alcanzar la meta prevista para el año 2010 ( $<10$ casos por 100.000 habitantes $)^{2}$.

El descenso de la tasa de casos pulmonares nuevos con baciloscopía positiva, que baja de 7,3 en el 2005, a 6,3 en el 2006, también contribuye a esta estimación optimista (Figura 1).

La distribución por sexo es de predominio masculino y la edad de los casos se desplaza hacia los mayores, lo que es propio de la situación epidemiológica característica de la declinación en países de baja prevalencia.

La desigualdad territorial persiste, manteniéndose las tasas más elevadas en las regiones extremas del país. Tarapacá y Magallanes nuevamente registran las mayores tasas, en tanto que las menores corresponden a los servicios de Aconcagua y Servicio Metropolitano Oriente.

Tabla 1. Morbilidad por Tuberculosis en todas sus formas y pulmonares con baciloscopia positiva. Chile, 1981 - 2005

\begin{tabular}{|c|c|c|c|c|}
\hline \multirow[t]{2}{*}{ Años } & \multicolumn{2}{|c|}{$\begin{array}{l}\text { TBC todas } \\
\text { las formas }\end{array}$} & \multicolumn{2}{|c|}{$\begin{array}{c}\text { TBC pulmonares } \\
\text { BK }(+)\end{array}$} \\
\hline & Número & Tasa* & Número & Tasa* \\
\hline 1981 & 7.337 & 65,0 & 4.519 & 39,9 \\
\hline 1985 & 6.645 & 55,0 & 3.500 & 28,9 \\
\hline 1989 & 6.728 & 51,9 & 3.216 & 24,9 \\
\hline 1994 & 4.138 & 29,5 & 1.951 & 12,1 \\
\hline 1999 & 3.420 & 22,8 & 1.497 & 10,0 \\
\hline 2003 & 2.908 & 18,4 & 1.283 & 8,1 \\
\hline 2004 & 2.794 & 17,5 & 1.317 & 8,3 \\
\hline 2005 & 2.546 & 15,7 & 1.186 & 7,3 \\
\hline
\end{tabular}

* Tasa por 100.000 habitantes; BK $(+)=$ Baciloscopia positiva.

\footnotetext{
* Programa Nacional de Control y Erradicación de la Tuberculosis (PROCET), Ministerio de Salud.

* Instituto Nacional del Tórax. Médico encargado del control de la Tuberculosis del Servicio de Salud Metropolitano Oriente.

*** Jefe Laboratorio de Micobacterias, Instituto de Salud Pública.
} 


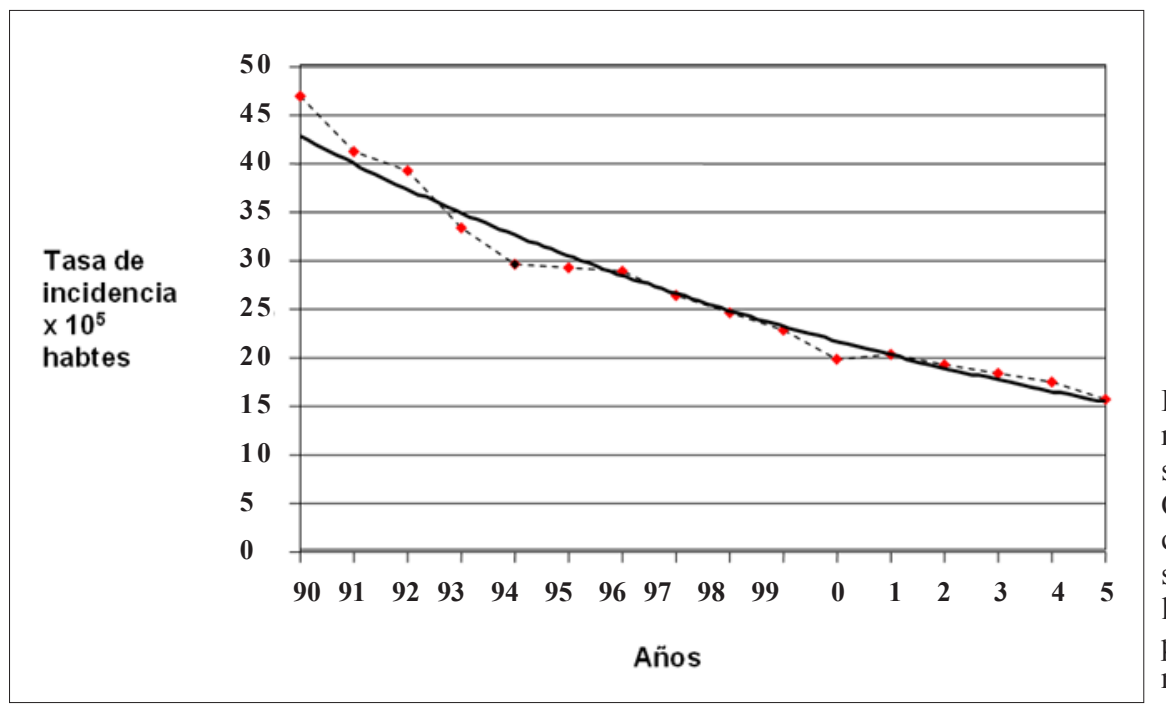

Figura 1. Evolución de la morbilidad por tuberculosis en todas sus formas. Chile, 1990-2005 La línea con rombos $(--)$ representa las tasas absolutas, la línea continua $(-)$ representa la tendencia exponencial.

En la Tabla 2, puede apreciarse el total de casos notificados, los casos nuevos TF y los casos nuevos pulmonares $\mathrm{BK}(+)$ en Chile hasta el tercer trimestre del año 2006 (para efectos de comparación de tasas se ha extrapolado este valor al año completo).

Para el análisis operacional y la destinación de recursos, los Servicios se han dividido en 4 grupos según sus tasas de tuberculosis TF. El grupo 1 tiene tasas $<10 / 100.000$ habitantes, es decir, según la OMS ha alcanzado la etapa de eliminación avanzada de la tuberculosis; el grupo 2 entre 10 y 20, significa haber superado el umbral de la etapa de eliminación de la tuberculosis; el grupo 3 entre 20 y 25, corresponde a aquellos Servicios que están cercanos a alcanzar el umbral de eliminación; y el grupo 4 $>$ 25/100.000 habitantes, aún no se acercan al umbral de la etapa de eliminación. La situación nacional proyectada para fines del 2006 es la señalada en la Tabla 3.

La tuberculosis extrapulmonar contribuye al total de casos registrados con más de un $24 \%$, situación que sólo es comparable con la de países desarrollados, que registran tasas de TBC totales menores de 20/100.000 habitantes.

Como un hecho que puede tener connotaciones de importancia para el control de la tuberculosis, se hace presente que la distribución por edades en el período 2002-2005, muestra una importante diferencia regional. La TBC infantil mantiene una mayor frecuencia en los Servicios con más altas tasas de TBC en la población general. En los Servicios de Arica e Iquique más del $15 \%$ de los casos se produce en menores de 20 años (en su gran mayoría en el subgrupo de 10 a 19 años), tres veces el promedio nacional que es de $6 \%$.

El análisis de los datos preliminares del año 2006 evidencia que el 42,3\% del país ya alcanzó la meta de eliminación avanzada (meta 2010). Es factible proyectar que un $48 \%$ adicional pueda alcanzarla antes del 2010. Menos de un 10\% de la población vive en Servicios con mayores dificultades para alcanzar la meta nacional ${ }^{2}$ (Tabla 3$)$.

\section{Situación operacional}

Para evaluar el cumplimiento de la norma difundida por el Programa Nacional de Control de la Tuberculosis (PROCET) de aumentar al menos en un 5\% las baciloscopías de diagnóstico, respecto del trienio anterior, decidimos presentar la evaluación del quinquenio 2001-2005. Estratificamos a los Servicios de Salud en cuatro grupos, según tasas de incidencia de 2005, promediamos las diferencias de cada año con su trienio precedente en cada grupo y lo expresamos en porcentaje (Figura 2). De acuerdo a recomendaciones de la Organización Panamericana de la Salud se utilizó gráfico con intervalos de confianza, dentro de cuyos límites se incluyen el $95 \%$ de las observaciones. Se observa que todos los grupos bajaron sus baciloscopías diagnósticas en $-0,7 \%$ como promedio. Llama la atención que al estratificarlos, los Servicios con tasas de tuberculosis más elevadas (grupos 3 y 4 ), aumentaron en un $5 \%$ y $2 \%$ respectivamente sus baciloscopías diagnósticas en el quinquenio. En cambio, los Servicios de menor pre- 
Tabla 2. Morbilidad de TBC por Servicios. Chile 2006. Total de casos notificados, nuevos en todas las formas y nuevos pulmonares baciloscopia+

\begin{tabular}{|c|c|c|c|c|c|c|c|}
\hline \multirow[t]{2}{*}{ Servicio de Salud } & \multirow[t]{2}{*}{$\begin{array}{c}\text { Población } \\
2006\end{array}$} & \multicolumn{2}{|c|}{$\begin{array}{l}\text { Casos notificados } \\
\text { tuberculosis todas formas }\end{array}$} & \multicolumn{2}{|c|}{$\begin{array}{l}\text { Casos nuevos (incidencia) } \\
\text { as todas formas }\end{array}$} & \multicolumn{2}{|c|}{$\begin{array}{c}\text { Casos nuevos } \\
\text { pulmonares BK (+) }\end{array}$} \\
\hline & & Número & Tasa & Número & Tasa & Número & Tasa \\
\hline Arica & 189.692 & 71 & 37,4 & 67 & 35,3 & 37 & 19,5 \\
\hline Iquique & 286.105 & 99 & 34,6 & 99 & 34,6 & 60 & 21,4 \\
\hline Antofagasta & 547.933 & 61 & 11,1 & 57 & 10,4 & 29 & 5,3 \\
\hline Atacama & 272.402 & 64 & 23,5 & 60 & 22 & 25 & 9,2 \\
\hline Coquimbo & 677.300 & 63 & 9,3 & 54 & 8 & 26 & 3,8 \\
\hline Valparaíso & 464.825 & 65 & 14 & 59 & 12,7 & 11 & 2,4 \\
\hline Viña del Mar & 968.225 & 96 & 9,9 & 92 & 9,5 & 38 & 3,9 \\
\hline Aconcagua & 248.955 & 16 & 6,4 & 15 & 6 & 4 & 1,6 \\
\hline Metropolitano Norte & 738.221 & 130 & 17,6 & 122 & 16,5 & 57 & 7,7 \\
\hline Metrop. Occidente & 1.118 .245 & 194 & 17,3 & 180 & 16,1 & 78 & 7 \\
\hline Metrop. Central & 1.036 .897 & 159 & 15,3 & 140 & 13,5 & 74 & 7,1 \\
\hline Metrop. Oriente & 1.170 .981 & 71 & 6,1 & 63 & 5,4 & 30 & 2,6 \\
\hline Metrop. Sur & 1.064 .413 & 192 & 18 & 170 & 16 & 87 & 8,2 \\
\hline Metrop. Sur Oriente & 1.479 .048 & 127 & 8,6 & 113 & 7,6 & 57 & 3,9 \\
\hline Lib. Bdo. O'Higgins & 849.120 & 86 & 10,1 & 82 & 9,7 & 24 & 2,8 \\
\hline Maule & 975.244 & 96 & 9,8 & 89 & 9,1 & 53 & 5,4 \\
\hline Nuble & 455.440 & 96 & 21,1 & 95 & 20,9 & 46 & 10,1 \\
\hline Concepción & 616.596 & 129 & 20,9 & 114 & 18,5 & 59 & 9,6 \\
\hline Arauco & 165.735 & 31 & 18,7 & 28 & 16,9 & 13 & 7,8 \\
\hline Talcahuano & 364.524 & 82 & 22,5 & 72 & 19,8 & 36 & 9,9 \\
\hline Bío Bío & 380.354 & 29 & 7,6 & 24 & 6,3 & 14 & 3,7 \\
\hline Araucanía Norte & 200.954 & 22 & 10,9 & 19 & 9,5 & 10 & 5 \\
\hline Araucanía Sur & 736.305 & 130 & 17,7 & 118 & 16 & 50 & 6,8 \\
\hline Valdivia & 373.712 & 49 & 13,1 & 45 & 12 & 20 & 5,4 \\
\hline Osorno & 231.669 & 54 & 23,3 & 54 & 23,3 & 32 & 13,8 \\
\hline Llanchipal & 562.860 & 85 & 15,1 & 77 & 13,7 & 33 & 5,9 \\
\hline Aysén & 100.417 & 17 & 16,9 & 14 & 13,9 & 7 & 7 \\
\hline Magallanes & 156.502 & 48 & 30,7 & 42 & 26,8 & 26 & 16,6 \\
\hline Total & 16.432 .674 & 2.362 & 14,4 & 2.164 & 13,2 & 1.036 & 6,3 \\
\hline
\end{tabular}

Tabla elaborada en base a información (Registro Nacional) hasta el tercer trimestre de 2006 y extrapolación para obtener una cifra anual tentativa. BK $(+)$ : baciloscopia positiva.

Tabla 3. Distribución por estratos según incidencia de TBC, 2006

\begin{tabular}{lcccccc}
\hline & $\begin{array}{c}\text { Tasa } \\
\mathbf{1 0 0 . 0 0 0}\end{array}$ & Población & $\begin{array}{c}\text { \% } \\
\text { Población }\end{array}$ & $\begin{array}{c}\text { Tasa Notificados } \\
\text { T. F. }\end{array}$ & $\begin{array}{c}\text { Tasa Notificados } \\
\text { Nuevos T. F. }\end{array}$ & $\begin{array}{c}\text { Tasa Pulmonar } \\
\text { Nuevos BK(+) }\end{array}$ \\
\hline Grupo I & $<10$ & 6.950 .181 & 42,3 & 8,7 & 7,9 & 3,7 \\
Grupo II & $10-19$ & 7.890 .683 & 48,0 & 16,8 & 15,2 & 7,0 \\
Grupo III & $20-25$ & 959.511 & 5,8 & 22,3 & 21,8 & 10,7 \\
Grupo IV & $>25$ & 632.299 & 3,9 & 34,5 & 32,9 & 19,5 \\
\hline
\end{tabular}

TF: todas las formas; $\mathrm{BK}(+)$ : baciloscopia positiva. 


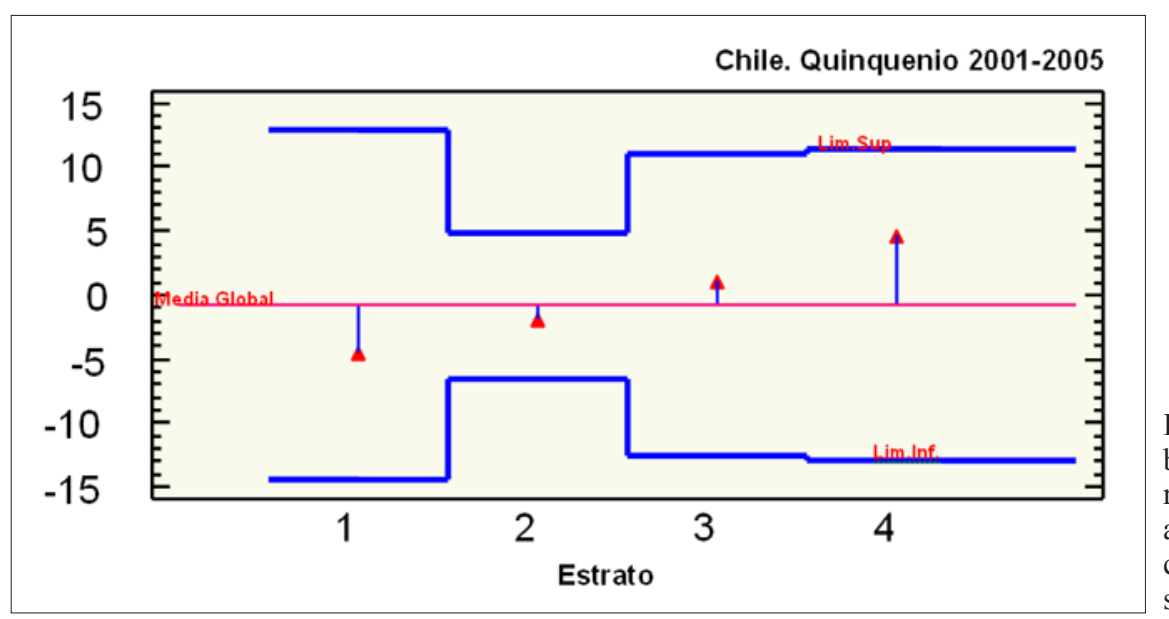

Figura 2. Variación de baciloscopías diagnósticas respecto del trienio móvil anterior. Estrato: agrupación de Servicios de Salud según tasa de TBC.

valencia disminuyeron las baciloscopías diagnósticas en $-2 \%$, los del grupo 2 (que incluye una población aproximada de 9 millones de habitantes) y en $-5 \%$ los del grupo 1 .

Estos resultados deben inducirnos a agilizar la difusión e implementación de las estrategias de focalización de la pesquisa en grupos de riesgo.

Respecto de los cultivos diagnósticos, todos los laboratorios de la red pública cumplen y superan la norma de cultivar al menos una de las dos baciloscopías diagnósticas, hecho relevante en la actual situación de la endemia. Es prioritario difundir esta estrategia en el ámbito de la medicina privada, dado que no es suficientemente conocida ${ }^{3}$.

Si se realiza un análisis desglosado por Servicio en relación a pesquisa, número de baciloscopías y número de baciloscopías necesarias para diagnosticar un caso entre 2004 y 2005 (Tabla 4), queda en claro que es necesario aumentar las solicitudes de baciloscopías diagnósticas. En tal sentido el apoyo de los Servicios de Urgencia y Servicios de Atención Primaria de Urgencia (SAPU) en general ha sido pobre. Recientemente se está implementando una nueva estrategia, apoyada en otro exitoso programa, el Programa de Enfermedades Respiratorias del Adulto (ERA), que ha habilitado un número creciente de salas en Centros de Salud de Atención Primaria, dotándolas de infraestructura diagnóstica y terapéutica de buen nivel y, principalmente, de recursos humanos adecuados, entre los que destacan kinesiólogos y enfermeras, quienes anualmente y en forma regular reciben capacitación.

El Programa de Tuberculosis participa en la capacitación de estos equipos en la patología que nos concierne. Esperamos que la pesquisa a nivel primario se beneficie de este programa.

Por otra parte, el análisis de la morbilidad y la progresiva caída en las tasas de tuberculosis, demuestra que la solicitud de baciloscopía en los sintomáticos respiratorios que consultan a los Servicios de Salud tendrá cada vez un menor rendimiento. Los enfermos cada vez se concentran más en grupos de riesgo bien definidos: adultos mayores, indigentes, reclusos, portadores de VIH, extranjeros de países de alta prevalencia e inmunodeprimidos, entre otros. Es por este motivo que el Programa de Control de la Tuberculosis durante los últimos años está diseñando estrategias para intensificar la pesquisa en estos grupos ${ }^{4}$.

Los resultados del tratamiento en la cohorte de los tuberculosos bacilíferos nunca antes tratados (Tabla 5, datos extraoficiales), muestra que de un total de 2.546 enfermos de tuberculosis en todas sus formas diagnosticados el año 2005, 1.296 tuvieron baciloscopía positiva (50,9\% del total). Este índice es útil para apreciar la tendencia hacia la eliminación de la enfermedad, puesto que los casos bacilíferos tienden a disminuir en regiones con bajas tasas. De hecho, el grupo $1(<10 / 100.000$ habitantes $)$ tuvo un $46,5 \%$ de casos pulmonares bacilíferos, en tanto que el grupo 4 (> 25/100.000 habitantes.) tuvo un $64,2 \%$ (destacando Magallanes donde la proporción alcanza al 74\%).

Se tabuló la cohorte de los pacientes vírgenes a tratamiento, que recibieron el esquema primario. De los 1.296 bacilíferos susceptibles de ingresar a la cohorte deben excluirse los antes tratados, aquellos que no recibieron el esquema primario, ya sea por toxicidad, por patología de base que lo impidiera o por otras causas (165 
casos). Como no se obtuvo información del resultado de la cohorte de los Servicios de Coquimbo (36 casos) y de Araucanía Norte (19 casos), finalmente entraron al análisis 1095 casos. En la Tabla 4 podemos apreciar el resultado preliminar de esta cohorte. En la primera columna se señala el número de pacientes aportados por cada Servicio y, por razones de espacio, la condición de egreso se expresa sólo en porcentajes.

El análisis de la condición de egreso de los pacientes ingresados a la cohorte evidencia una bajísima tasa de fracasos del tratamiento, lo que traduce una buena organización en la aplicación de la estrategia DOTS (tratamiento abreviado supervisado). La alta tasa de letalidad de 9,2\% (meta OMS $<3 \%$ ) es la que determina en mayor medida el no alcanzar la meta de la OMS de un $85 \%$ de curación, la que alcanza solo al $82,7 \%$. La letalidad, desgraciadamente incluye casos de pacientes con tuberculosis que no fallecen de esta causa, sin embargo hay evidencias que también influye el diagnóstico tardío. Se puede observar en la Tabla 5, una amplia variación en los diferentes Servicios, lo que amerita un esfuerzo a nivel central para determinar los factores involucrados en cada lugar. Sabemos, por ejemplo, que en los Servicios Metropolitano Sur y Oriente la letalidad se asocia a la situación de adulto mayor.

La condición de abandono alcanza al 6,2\%, cifra superior a la meta OMS $(<5 \%)$. Esta cifra

Tabla 4. Número de baciloscopías de pesquisa y número de baciloscopías necesarias para diagnosticar un caso. Chile 2004-2005

\begin{tabular}{|c|c|c|c|c|c|c|}
\hline \multirow[t]{2}{*}{ Servicio de Salud } & \multicolumn{2}{|c|}{ Número BK diagnósticas } & \multirow{2}{*}{$\begin{array}{c}\text { Diferencia } \\
\%\end{array}$} & \multicolumn{2}{|c|}{ Número BK para dg. 1 caso } & \multirow{2}{*}{$\begin{array}{c}\text { Diferencia } \\
\%\end{array}$} \\
\hline & 2004 & 2005 & & 2004 & 2005 & \\
\hline Arica & 10.676 & 9.960 & $-6,7$ & 152 & 151,5 & $-0,3$ \\
\hline Iquique & 6.957 & 6.209 & $-10,8$ & 131 & 127 & $-3,1$ \\
\hline Antofagasta & 7.454 & 5.175 & $-30,6$ & 199 & 216 & 8,5 \\
\hline Atacama & 5.388 & 6.454 & 19,8 & 150 & 280 & 86,7 \\
\hline Coquimbo & 9.774 & 7.818 & $-20,0$ & 132 & & \\
\hline Valparaíso & 12.395 & 11.526 & $-7,0$ & 188 & 235 & 25,0 \\
\hline Viña del Mar & 12.122 & 10.373 & $-14,4$ & 209 & 253 & 21,1 \\
\hline Aconcagua & 2.677 & 2.034 & $-24,0$ & 191 & 290 & 51,8 \\
\hline Metropolitano Norte & 15.696 & 13.497 & $-14,0$ & 162 & 138 & $-14,8$ \\
\hline Metrop. Occidente & 23.141 & 21.732 & $-6,1$ & 187 & 205 & 9,6 \\
\hline Metrop. Central & 15.230 & 12.944 & $-15,0$ & 169 & 130 & $-23,1$ \\
\hline Metrop. Oriente & 15.954 & 14.105 & $-11,6$ & 183 & 143 & $-21,9$ \\
\hline Metrop. Sur & 29.992 & 27.675 & $-7,7$ & 256 & 237 & $-7,4$ \\
\hline Metrop. Sur Oriente & 25.907 & 26.323 & 1,6 & 285 & 318 & 11,6 \\
\hline Lib. Bdo. O'Higgins & 12.178 & 8.332 & $-31,6$ & 203 & 104 & $-48,8$ \\
\hline Maule & 16.482 & 15.187 & $-7,9$ & 206 & 143 & $-30,6$ \\
\hline Ñuble & 10.921 & 10.074 & $-7,8$ & 331 & 206 & $-37,8$ \\
\hline Concepción & 14.629 & 16.524 & 13,0 & 164 & 261 & 59,2 \\
\hline Arauco & 5.359 & 4.186 & $-21,9$ & 357 & 246 & $-31,1$ \\
\hline Talcahuano & 9.351 & 8.709 & $-6,9$ & 176 & 350 & 98,9 \\
\hline Bío Bío & 9.073 & 12.530 & 38,1 & 394 & 108 & $-72,6$ \\
\hline Araucanía Norte & 4.861 & 3.235 & $-33,5$ & 211 & & \\
\hline Araucanía Sur & 15.945 & 12.841 & $-19,5$ & 295 & 238 & $-19,3$ \\
\hline Valdivia & 7.082 & 6.092 & $-14,0$ & 157 & 185 & 17,8 \\
\hline Osorno & 7.816 & 7.164 & $-8,3$ & 206 & 217 & 5,3 \\
\hline Llanchipal & 5.172 & 5.736 & 10,9 & 165 & 112 & $-32,1$ \\
\hline Aysén & 2.196 & 1.835 & $-16,4$ & 220 & 405 & 84,1 \\
\hline Magallanes & 4.575 & 2.961 & $-35,3$ & 208 & 92 & $-55,8$ \\
\hline Total & 308.472 & 291.231 & $-5,6$ & 202 & 172 & $-14,9$ \\
\hline
\end{tabular}


es difícil de modificar puesto que los centros de atención primaria no cuentan con recursos para ir al rescate de pacientes que dejan de asistir y, por otra parte, hay situaciones como la de los Servicios Metropolitano Sur y Central que manejan una alta proporción de indigentes y reclusos, sin los medios para el adecuado seguimiento de ellos. De ahí que sean los Servicios con mayor densidad de población los que tienen más problemas: el Servicio Sur la mayor tasa de abandono del país y el Central el mayor número de traslados sin conocer su condición de término de tratamiento.

El estudio de contactos de casos índices de tuberculosis bacilífera, tiene muy alto rendimiento. Si en el país, entre los sintomáticos respiratorios (SR) necesitamos hacer alrededor de 200 baciloscopías para encontrar un caso, entre los contactos sólo necesitamos 78. Este grupo tiene considerable mayor riesgo de padecer tuberculosis que la población general.

En la Tabla 6, aunque con registros incompletos, se aprecia una amplia dispersión en el número de contactos por caso de tuberculosis bacilífera. No deja de ser curioso que en Arica y Atacama, con tan baja densidad de población, sea mayor número de contactos, casi el doble, que en la Región Metropolitana. Por otra parte, parece raro que en Concepción cada caso de tuberculosis conviva con sólo una persona.

Es encomiable el Servicio de Aconcagua, que con tasas tan bajas de tuberculosis, se ha abocado con gran éxito al estudio de esta población en forma amplia, con casi 7 contactos por caso,

Tabla 5. Condición de egreso de la cohorte de pacientes bacilíferos con esquema primario (expresada en porcentajes)

\begin{tabular}{|c|c|c|c|c|c|c|}
\hline Servicio de Salud & Total pctes. & Curados & Fracaso & Fallecido & Abandono & Traslado \\
\hline Arica & 49 & 88 & 2 & 4 & 2 & 4 \\
\hline Iquique & 49 & 77,5 & 0 & 4,1 & 14,3 & 4,1 \\
\hline Antofagasta & 42 & 74 & 0 & 16,7 & 9,3 & 0 \\
\hline Atacama & 18 & 83,2 & 0 & 5,6 & 5,6 & 5,6 \\
\hline \multicolumn{7}{|l|}{ Coquimbo } \\
\hline Valparaíso & 43 & 90,7 & 0 & 7 & 2,3 & 0 \\
\hline Viña del Mar & 40 & 85 & 0 & 15 & 0 & 0 \\
\hline Aconcagua & 4 & 100 & 0 & 0 & 0 & 0 \\
\hline Metrop. Norte & 50 & 84 & 2 & 4 & 10 & 0 \\
\hline Metrop. Occidente & 79 & 81 & 0 & 12,7 & 6,3 & 0 \\
\hline Metrop. Central & 77 & 74 & 0 & 7,8 & 7,8 & 10,4 \\
\hline Metrop. Oriente & 33 & 81,7 & 0 & 6,1 & 6,1 & 6,1 \\
\hline Metrop. Sur & 84 & 76,2 & 0 & 10,7 & 13,1 & 0 \\
\hline Metrop. Sur Oriente & 65 & 89 & 0 & 7 & 3 & 0 \\
\hline Lib. Bdo. O'Higgins & 64 & 81,3 & 0 & 14 & 3,1 & 1,6 \\
\hline Maule & 50 & 80 & 0 & 16 & 4 & 0 \\
\hline Ñuble & 47 & 87,3 & 0 & 8,5 & 2,1 & 2,1 \\
\hline Concepción & 60 & 88,3 & 0 & 5 & 6,7 & 0 \\
\hline Arauco & 16 & 100 & 0 & 0 & 0 & 0 \\
\hline Talcahuano & 20 & 95 & 0 & 5 & 0 & 0 \\
\hline Bío Bío & 14 & 86 & 0 & 7 & 7 & 0 \\
\hline \multicolumn{7}{|l|}{ Araucanía Norte } \\
\hline Araucanía Sur & 52 & 92,3 & 0 & 5,8 & 0 & 1,9 \\
\hline Valdivia & 20 & 85 & 0 & 5 & 10 & 0 \\
\hline Osorno & 37 & 81 & 0 & 13,5 & 5,5 & 0 \\
\hline Llanchipal & 54 & 81,5 & 0 & 7,4 & 11,1 & 0 \\
\hline Aysén & 4 & 75 & 0 & 25 & 0 & 0 \\
\hline Magallanes & 24 & 70,8 & 0 & 25 & 4,2 & 0 \\
\hline Total & 1.095 & 82,7 & 0,2 & 9,2 & 6,2 & 1,7 \\
\hline
\end{tabular}


Tabla 6. Estudio de contactos: Cobertura y rendimiento

\begin{tabular}{|c|c|c|c|c|c|c|c|}
\hline Servicio de Salud & Casos & $\begin{array}{c}\text { Contactos } \\
\text { por caso }\end{array}$ & $\begin{array}{l}\text { Censados } \\
\text { (n) }\end{array}$ & $\begin{array}{l}\text { Estudiados } \\
\text { (n) }\end{array}$ & $\begin{array}{c}\text { Contactos } \\
\text { estudiados } \\
(\%)\end{array}$ & Casos TBC & $\underset{(\%)}{\text { Rendimiento }}$ \\
\hline Arica & 61 & 9,64 & 588 & 511 & 86,90 & 4 & 0,78 \\
\hline Iquique & & & & & 97,00 & & 2,90 \\
\hline Antofagasta & 43 & 2,14 & 92 & 81 & 88,04 & 1 & 1,23 \\
\hline Atacama & 18 & 6,50 & 117 & 114 & 97,44 & 2 & 1,75 \\
\hline \multicolumn{8}{|l|}{ Coquimbo } \\
\hline Valparaíso & 50 & 4,06 & 203 & 199 & 98,03 & 3 & 1,51 \\
\hline Viña del Mar & 61 & & & & & & \\
\hline Aconcagua & 7 & 6,57 & 46 & 46 & 100,00 & 2 & 4,35 \\
\hline \multicolumn{8}{|l|}{ Metropolitano Norte } \\
\hline Metrop. Occidente & 129 & 4,6 & 598 & 539 & 90,1 & 3 & 0,6 \\
\hline Metrop. Central & 107 & 3,22 & 345 & 252 & 73,04 & 5 & 1,98 \\
\hline Metrop. Oriente & 58 & 2,26 & 131 & 123 & 93,89 & 2 & 1,63 \\
\hline Metrop. Sur & 113 & 3,50 & 396 & 354 & 89,39 & 2 & 0,56 \\
\hline Metrop. Sur Oriente & 83 & 4,31 & 358 & 340 & 94,97 & 8 & 2,35 \\
\hline Lib. Bdo. O'Higgins & 70 & 4,30 & 301 & 299 & 99,34 & 3 & 1,00 \\
\hline Maule & 55 & 3,16 & 174 & 169 & 97,13 & 0 & 0,00 \\
\hline \multicolumn{8}{|l|}{ Ñuble } \\
\hline Concepción & 129 & 1,16 & 149 & 104 & 69,80 & 0 & 0,00 \\
\hline Arauco & 21 & 3,67 & 77 & 71 & 92,21 & 1 & 1,41 \\
\hline Talcahuano & 24 & 3,79 & 91 & 80 & 87,91 & 0 & 0,00 \\
\hline Bío Bío & 11 & & & & 99,00 & & \\
\hline \multicolumn{8}{|l|}{ Araucanía Norte } \\
\hline Araucanía Sur & 101 & 4,19 & 423 & 377 & 89,13 & 7 & 1,86 \\
\hline Valdivia & 52 & & & & & & \\
\hline Osorno & 62 & 2,58 & 160 & 159 & 99,38 & 2 & 1,26 \\
\hline Llanchipal & 54 & & & & 100,00 & 0 & 0 \\
\hline Aysén & 4 & & & & 100,00 & 0 & 0 \\
\hline \multicolumn{8}{|l|}{ Magallanes } \\
\hline Total & 1.184 & 3,08 & 3.651 & 3.279 & 89,81 & 42 & 1,28 \\
\hline
\end{tabular}

estudiándolos a todos con excelente rendimiento (4,35\% de enfermos). Mejores empresas han sido las de los Servicios Metropolitano Sur Oriente y Libertador B. O'Higgins, que nos han demostrado lo que rinde una buena cobertura, de sobre el $99 \%$ del estudio de contactos, asociado en los Servicios Metropolitano Sur Oriente e Iquique, a un alto rendimiento $(2,35$ y $2,9 \%$ respectivamente de nuevos enfermos).

\section{Análisis y conclusiones}

Las reformas de la salud han significado, para el Programa de Control de la Tuberculosis, una reestructuración con algunos costos. Se han desarmado equipos técnicos, destinando horas antes asignadas a tuberculosis a otras funciones.

Existe la necesidad de capacitar profesionales en forma continua en una enfermedad que requiere rigurosidad en el manejo y registro y que al ir acercándose a la etapa de eliminación avanzada, necesita de un diagnóstico de situación más preciso y de nuevas herramientas. La ley de autoridad sanitaria determina la existencia de un responsable de tuberculosis a nivel de la SEREMI. Estos profesionales, también requieren de una capacitación específica destinada a lograr una coordinación extra-sectorial para focalizar la pesquisa y el tratamiento en los gru- 
pos de alto riesgo, incluyendo una acción coordinada en penitenciarías, hogares de ancianos, hospederías, residencias con hacinamiento, que suelen recibir extranjeros provenientes de zonas de mayor prevalencia de tuberculosis y, en general, todos los grupos especiales que detecte cada Servicio. Estas acciones requieren de una gestión intersectorial cuyas herramientas necesitan ser creadas. En tal sentido, un acuerdo marco con gendarmería es una iniciativa paradigmática a concretarse en breve ${ }^{5}$.

\section{Propuestas de estrategias para 2007}

1. Es prioritaria, como siempre, la educación continua en todos los niveles. Para ello siguen siendo necesarios recursos y condiciones favorables de los Servicios para apoyar esta tarea.

2. Hay que completar el diagnóstico de situación de los grupos de riesgo de hacer tuberculosis. Algunos Servicios los tienen bien caracterizados y están desarrollando estrategias específicas en tal sentido, pero otros carecen de recursos humanos y técnicos para dedicarse a esta tarea y requerirán apoyo del Nivel Central y de la SEREMI.

3. Está pendiente a nivel nacional el análisis de la cohorte de pacientes antes tratados. El estudio de la condición de ingreso y egreso de estos pacientes al tratamiento y la tabulación de su patrón de resistencia a fármacos antituberculosos, nos permitirá elaborar normas más precisas para evitar recaídas y detectar los casos con factores de riesgo de recaer o fracasar.
4. Es necesario aumentar las investigaciones operacionales en tuberculosis. Sería útil determinar, con nuevas técnicas, el riesgo de infección en diferentes zonas de alta, media y baja prevalencia. Esto nos permitiría fundamentar cambios de estrategia en la pesquisa y focalizar la acción en determinados grupos, así como ampliar el estudio de contactos y planificar una segunda reevaluación.

5. También es una tarea pendiente estudiar la posibilidad de incluir las nuevas herramientas que la biología molecular está desarrollando para el control de la tuberculosis, no sólo en la implementación de diagnósticos más oportunos sino, especialmente, para estudios de epidemiología molecular ${ }^{6}$.

\section{Bibliografía}

1.- PROCET. Tuberculosis en Chile. Programa Nacional de Control y Erradicación de la Tuberculosis (PROCET) 2005-2006. Ministerio de Salud, Chile.

2.- ZÚÑIGA M. Situación de la Tuberculosis en Chile 2002 y su posible evolución. Rev Chil Enf Respir 2003; 19 : 179-91.

3.- GONZÁLEZ P. Optimización del diagnóstico microbiológico en infecciones por micobacterias. Rev Chil Enf Respir 2004; 20: 182-5.

4.- FARGA V. Hacia la erradicación de la tuberculosis. Rev Chil Enf Respir 2006; 22: 55-67.

5.- FARGA V. Resumen de actividades Comité de Tuberculosis. Región Metropolitana 2005. Rev Chil Enf Respir 2006; 22: 127-34.

6.- MARCONE P. Aplicaciones de la biología molecular en el Programa Nacional de Tuberculosis en Chile: ¿Lujo o necesidad?. Rev Chil Enf Respir 2006; 22: 201-8. 